\title{
Los probióticos: alimentos funcionales para lactantes
}

\author{
Marbe Alexandra Cardona-Arengas* \\ Beatriz Estella López-Marín**
}

\begin{abstract}
* Química. Especialista en Alimentación y Nutrición. Candidata a Msc. en Innovación Alimentaria y Nutrición. Corporación Universitaria Lasallista. Docente. Universidad de Antioquia. Medellín. Colombia.

**Nutricionista Dietista. Msc Ciencias Farmacéuticas y Alimentarias. PhD. Ciencias Farmacéuticas y Alimentarias. Docente. Universidad de Antioquia. Escuela de Nutrición y Dietética. Medellín. Colombia

Correspondencia: Doctora Marbe Alexandra Cardona. Dirección: Calle 57 \# 68C-65 torre 2A apto 622. Unidad cerrada Peña verde. Bello (Antioquia). Correo electrónico: alexacarengas@gmail.com. Celular 3147901236
\end{abstract}

\section{Resumen}

La nutrición y salud de los lactantes es de vital importancia en la sociedad actual. Los probióticos surgen como suplementos para la innovación de alimentos funcionales para lactantes. El objetivo de esta revisión fue conocer cuáles probióticos son los más empleados en el desarrollo de nuevos productos alimenticios funcionales para lactantes. Se contó con más de cincuenta referencias bibliográficas que tuvieran el desarrollo de alimentos funcionales a partir de una bacteria probiótica y sus beneficios en salud, publicadas en las siguientes bases de datos: EBSCO HOST, PLOS y DOAJ. Se encontró que el Bacillus coagulans surge como una alternativa que mejora las condiciones de biodisponibilidad, y se encontraron estudios que indagan por beneficios de los probióticos en el aspecto antropométrico. Reconociendo los beneficios de los probióticos en la salud de los lactantes, su aplicación exige mayor cumplimiento de criterios de la Sociedad Europea de Gastroenterología, Hepatología y Nutrición Pediátrica. MÉD.UIS.2019;32(2): 31-9

Palabras Clave: Lactante. Alimentos funcionales. Probiótico. Bacillus coagulans. Gastroenterología. Antropometría.

\section{Probiotics: functional foods for infants}

\section{Abstract}

The nutrition and infant's health is vitally important in today's society. The probiotics arise as supplements for the innovation of functional foods for infants. The objective of this review was to find out which probiotics are currently most used in the development of new functional food products for infants. We counted on more than fifty bibliographical references about the development of functional foods from a probiotic bacterium and its healthy benefits, published in the following databases: EBSCO HOST, PLOS and DOAJ. It was found that Bacillus Coagulans emerges as an alternative that improves bioavailability conditions, and studies that investigate the benefits of probiotics in anthropometric aspect. Recognizing the benefits of probiotics in the health of infants, its application requires greater compliance with criteria of the European Society for Pediatric Gastroenterology, Hepatology and Nutrition. MÉD.UIS.2019;32(2): 31-9

Keywords: Infant. Functional Food. Probiotics. Bacillus coagulans. Gastroenterology. Anthropometry.

¿Cómo citar este artículo?: Cardona-Arengas MA, López-Marín BE. Los probióticos: alimentos funcionales para lactantes. MÉD. UIS.2019;32(2): 31-9. doi: 10.18273/revmed.v32n2-2019004 


\section{Introducción}

Los probióticos se denominan como un amplio grupo de bacterias no patógenas, que presentan diversos beneficios potenciales para la salud, y que se vienen utilizando durante siglos en la forma de productos fermentados a base de leche, tales como yogur ${ }^{1,2,3}$, bebidas lácteas ${ }^{4,5}$, postres ${ }^{6}$, purés, quesos, entre otros ${ }^{7}$. La Organización Mundial de la Salud (OMS), ha definido a los probióticos como "microorganismos vivos que, cuando se administran en cantidades adecuadas, confieren un beneficio a la salud" ${ }^{2,8,9,10}$. La Academia Americana del Comité de Pediatría sobre Nutrición en Probióticos los definen como "los microbios que generan pequeños subproductos moleculares metabólicos que ejercen una influencia reguladora beneficiosa en las funciones biológicas y que pueden funcionar como inmunomoduladores" "1. Finalmente, una reciente declaración de Liu $X$ et al., definen a los probióticos como "un microorganismo vivo que como suplemento alimenticio es beneficioso para la salud"'2. Los probióticos se suplementan con prebióticos, los cuales son componentes alimentarios que aportan un beneficio en salud, asociado a un efecto en la microbiota ${ }^{13}$.

Uno de los aspectos encontrados, y al cual se le da una gran relevancia con el empleo de probióticos en alimentos, es que es denominado alimento funcional por generar un beneficio en salud ${ }^{13,15,16}, \mathrm{y}$ está relacionado con los requisitos que deben tener estos microorganismos benéficos: en preparados para seres humanos, por ejemplo, se requiere que el alimento tenga un mínimo de un millón de organismos probióticos viables por gramo de producto, o $10^{7}$ Unidades Formadoras de Colonia $g^{-1}$ (UFC), consumidos en cantidades suficientes que puedan producir una ingesta diaria de $10^{8} \mathrm{UFC}^{17,18,19}$. Otros de los requisitos son que los microorganismos deben ser de origen humano, no patógeno, resistentes a la destrucción por procesamiento térmico y por secreciones del tracto gastrointestinal, capaces de colonizarlo y de producir sustancias antimicrobianas, y de modular respuestas inmunes e influir en las actividades metabólicas humanas $13,14,20$. Todas las características deben ser cumplidas a cabalidad por el microorganismo que sea empleado en el desarrollo del producto alimenticio para consumo humano, pues un incumplimiento en alguna de estas puede generar efectos negativos en la persona, o no producir ninguno de los efectos benéficos esperados. Es así como la normatividad internacional tiene estipulada, en su reglamentación, seguir el documento denominado Guidelines for the Evaluation of Probiotics in Food de la Organización de las Naciones Unidas para la Alimentación y la Agricultura (FAO) y la OMS antes de solicitar un Health Claim para un producto alimenticio ${ }^{13}$. Este documento explica el paso a paso de todos los requisitos que debe cumplir un producto alimentario funcional con probióticos para inicialmente ser aceptado y ser evaluado como Health claim. Los requisitos que se deben cumplir en el alimento son:

1. Evaluación de la identidad y seguridad de los microorganismos probióticos.

2. Evaluación de la seguridad de los probióticos en los humanos.

3. Evaluación de la eficacia de los probióticos en la salud ${ }^{17,18}$.

Entre los entes más rigurosos que reglamentan el uso de probióticos en alimentos se encuentra la Europea Food Safety Authority, la cual exige varios estudios clínicos para poder aceptar un informe o documento escrito, que denominan dossier ${ }^{19}$. Mientras que para la EFSA no hay términos medios, las regulaciones en Japón, por ejemplo, permiten clasificar un alimento probiótico funcional en dos niveles: el tipo 1, que se denomina clasificado, y el tipo 2, que se denomina estandarizado, con solo haber cumplido el requisito

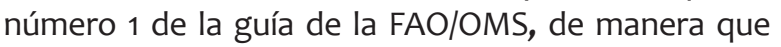
pueden ser comercializados. Casi lo mismo sucede en América con la FDA, lo cual aplica para todo el continente, en la cual la regulación permite vender un producto probiótico funcional categorizándolo solamente como nutracéutico ${ }^{20}$. Por todos los aspectos ya mencionados, el objetivo de este artículo es realizar una revisión detallada del estado del arte sobre el empleo de las bacterias probióticas, principalmente en aquellos que son los actualmente más empleados en el desarrollo de nuevos productos alimenticios funcionales para lactantes en el ámbito de la industria.

\section{Metodología}

Se contó con más de 50 referencias bibliográficas que tuvieran como característica los siguientes componentes: desarrollo de un nuevo producto funcional a partir de una bacteria probiótica y su efecto benéfico en salud primordialmente en lactantes, además de las normativas que vigilan estas innovaciones. Estas referencias bibliográficas 


\section{Mayo - Agosto}

se encontraron publicadas en bases de datos EBSCO HOST, PLOS y DOAJ, y se usaron las palabras claves: lactante, alimento funcional, probióticos, Bacillus coagulans, Gastroenterología y Antropometría. La búsqueda bibliográfica se llevó a cabo entre marzo de 2016 y octubre de 2017. Los criterios de inclusión implicaron a los artículos que estudiaban los efectos clínicos de los probióticos en lactantes y las innovaciones alimentarias funcionales para los mismos. Sin embargo, mediante este tipo de criterio, se pudo evidenciar que existe poca literatura científica reciente al respecto. Igualmente, se rechazaron aquellos artículos sobre probióticos relativos a los probióticos como aplicación en alimentación veterinaria $\mathrm{o}$ en procesos de descontaminación ambiental a partir de microorganismos. Se encontró que las matrices alimentarias más estudiadas son las de la leche y sus productos, mientras que, para productos no lácteos, los probióticos siguen siendo un nicho de estudio apenas explorado.

\section{Desarrollo del tema}

En los últimos años, el interés por el papel benéfico de las bacterias probióticas en la salud humana se ha intensificado de gran manera, debido a que mejoran el equilibrio microbiano intestinal ${ }^{21}$; estos beneficios en salud se atribuyen a la ingesta de alimentos que contienen cultivos probióticos ${ }^{8}$. Otros efectos benéficos que se les atribuyen son que: reducen o eliminan dolencias tales como la irritación del colon, el estreñimiento y la diarrea del viajero ${ }^{17,22}$; inhiben bacterias patógenas ${ }^{23}$; sintetizan vitaminas del grupo B, reducen los niveles de amoníaco en la sangre y la absorción del colesterol24; evitan la formación de tumores ${ }^{25}$, mejoran la absorción de calcio y la utilización de lactosa mediante la producción de b-galactosidasa ${ }^{26,27}$, contribuyen con efectos benéficos en infecciones gastrointestinales; disminuyen la inflamación en la enfermedad intestinal inflamatoria y en las afecciones del hígado ${ }^{10}$; mejoran la sintomatología en la diabetes ${ }^{28}$; contribuyen con una disminución de peso y con el tratamiento contra la obesidad ${ }^{29}$, y se ha visto, además, que combaten la infección por Helicobacter pylori ${ }^{30,31}$.

Para comprobarse los efectos benéficos de estos microorganismos, son necesarios estudios clínicos que validen su efecto, aspecto considerado en la normatividad de la FAO/OMS, la cual es el punto de partida para cualquier otra reglamentación o regulación al respecto en el mudo ${ }^{17}$.

Entre los requisitos que debe cumplir un probiótico está el de poblar el tracto gastrointestinal; algunos estudios solo han hipotetizado al respecto, como el de Hickey et al., mientras que otros ya han empezado a incurrir en estos hallazgos, y han demostrado que ciertas cepas como las de Lactobacillus y Bifidobacterium, sí tienen la capacidad de poblar este tracto. Por ejemplo en el estudio realizado por Rodes et al.; donde mediante un análisis in vitro se monta una simulación de la microbiota dentro de un colon 32,33,34,35.

Es de anotar que algunos ensayos clínicos con probióticos se han realizado en población infantil, donde se ha encontrado que una mayor densidad de bacterias beneficiosas en los bebés logra conferir un mayor grado de protección contra enfermedades, tales como alergias e infecciones gastrointestinales ${ }^{23,27,36,37,38}$. Asimismo, se ha encontrado que la colonización bifidobacteriana en población lactante está asociada a un menor riesgo de padecer alergias ${ }^{21,22,24,30}$, entre ellas la rinitis $^{25}$. Además, se han reportado estudios sobre tratamientos en la enterocolitis necrotizante, una de las enfermedades más características de los recién nacidos y que tiene alto riesgo de muerte ${ }^{11,26,28}$.

\section{Probióticos más utilizados en la industria de alimentos funcionales}

La literatura científica recolectada reporta las Bacterias Acido Lácticas (BAL) como una de las especies más comúnmente utilizadas como probióticos en la industria de alimentos funcionales. Entre ellas se destacan dos de gran importancia y empleo en la industria de alimentos, Lactobacillus y Bifidobacterium ${ }^{2,21,29,31}$, pues son bacterias cuyas cepas se encuentran principalmente en el tracto gastrointestinal humano del adulto ${ }^{39}$; un alimento natural que las contiene en gran cantidad es la leche materna humana. Estas bacterias probióticas se emplean en procesos de fermentación u obtención de otras sustancias que impliquen la producción de un ingrediente funcional especial, para conseguir el efecto nutricional y beneficioso en la salud ${ }^{3,38}$. Desde el punto de vista normativo, estos probióticos cuentan con el respaldo de la FAO/OMS Guidelines for the Evaluation of Probiotics in Food, ya que cumplen con los tres numerales de requisitos exigidos por este ${ }^{17,15}$. 


\section{Género Lactobacillus y Bifidobacterium}

El Lactobacillus es un género muy heterogéneo que abarca bacterias con una amplia gama de propiedades bioquímicas y fisiológicas ${ }^{23}$. Es el más grande de los que pertenecen a la familia BAL (Ver Tabla 1), con 185 especies descritas de forma válida en la literatura, y que aumentaron de forma sustancial en 145, en 2008, como consecuencia de la reclasificación de otras múltiples especies. Desde la clasificación inicial de la especie Lactobacillus acidophilus, en 1920 (Holanda), hasta alrededor de 1970, muchos Lactobacillus aislados de las superficies mucosas humanas se siguieron identificando colectivamente como $\mathrm{L}$. acidophilus. ${ }^{\circ}$.

El L. acidophilus es una bacteria Gram positiva, crece de manera óptima desde 37 hasta $42^{\circ} \mathrm{C}$, y máximo $45^{\circ} \mathrm{C}$. La especie alcanza su mayor tasa de crecimiento en medios ligeramente ácidos de $\mathrm{pH} 5,5$ a 6,0, y cesa su crecimiento por debajo de $\mathrm{pH} 4,0$. Es un fermentador obligatorio en la producción de ácido láctico a partir de la fermentación de hidratos de carbono, y es uno de los más sensibles al oxígeno". La caracterización de sus cepas probióticas ha permitido estudiar cuanto abarca su estabilidad en producto, su resistencia a la bilis, su tolerancia a bajo $\mathrm{pH}$, su adhesión a los colonocitos humanos en cultivo celular, su producción antimicrobiana y su actividad lactasa ${ }^{21,32,41,42}$

Otra BAL muy caracterizada en sus efectos benéficos son los Enterococos, bacterias importantes en los alimentos y en la microbiología clínica ${ }^{43}$. Son bacterias Gram positivas, sin esporulación, formadoras de catalasa-oxidasa con carga negativa, y son bacterias facultativas anaerobias que se pueden producir solas, en parejas o en cadenas. La mayoría de las cepas de esta especie son comensales inofensivas y mayormente presentan carácter benéfico para la salud. Además, se pueden utilizar en cultivos iniciadores para alimentos fermentados; por ejemplo, Enterococcus faecalis se utiliza con éxito en la preparación de quesos, acelerando la maduración y mejorando las características organolépticas de éstos. A raíz de esto, se intensifica el interés tecnológico por los Enterococos, los cuales se utilizan como el cultivo iniciador propicio para diferentes quesos europeos, tales como Mozarella Water Buffalo, Feta, Venaco y el queso Cebreiro. Finalmente, debido a las propiedades bioquímicas deseables y bien documentadas de esta bacteria, tales como la capacidad de producir péptidos antimicrobianos conocidos como bacteriocinas, puede ser utilizada como biopreservativo en alimentos ${ }^{44}$.

Tabla 1. Géneros de Lactobacillus y Bifidobacterium reconocidos como probióticos

\begin{tabular}{|c|c|}
\hline Género Lactobacillus & Género Bifidobacterium \\
\hline Lactobacillus acidophilus & B. adolescentis \\
\hline Lactobacillus johnsonii & B. angulatum \\
\hline Lactobacillus paracasei & B. animalis \\
\hline Lactobacillus casei & B. asteroides \\
\hline Lactobacillus rhamnosus & B. dum BIFI \\
\hline Lactobacillus plantarum & B. boum \\
\hline $\begin{array}{l}\text { Lactobacillus delbrueckii } \\
\text { subsp bulgaricus }\end{array}$ & B. breve \\
\hline $\begin{array}{l}\text { Lactobacillus delbrueckii } \\
\text { subsp lactis }\end{array}$ & B. catenulatum \\
\hline Lactobacillus cellobiosus & B. choerinum \\
\hline Lactobacillus curvatus & B. coryneforme \\
\hline Lactobacillus fermentum & B. cuniculi \\
\hline Lactobacillus reuteri, & B. dentium \\
\hline Lactobacillus brevis & B. gallicum \\
\hline Lactobacillus salivarius & B. gallinarum \\
\hline Lactobacillus helveticus & B. indicum \\
\hline Lactobacillus amylovorus & B. longum \\
\hline Lactobacillus crispatus & B. magnum \\
\hline Lactobacillus gallinarum & B. merycicum \\
\hline Lactobacillus gasseri & B. mínimo \\
\hline Lactobacillus coryniformis & B. pseudocatenulatum \\
\hline Lactobacillus agilis & B. pseudolongum \\
\hline Lactobacillus aviarius & B. psychraerophilum \\
\hline Lactobacillus murinus & B. pullorum \\
\hline Lactobacillus hamsteri & B. ruminantium \\
\hline Lactobacillus intestinalis & B. saeculare \\
\hline \multirow[t]{4}{*}{ Lactobacillus ruminis } & B. scardovii \\
\hline & B. sutil \\
\hline & B. thermacidophilum \\
\hline & B. thermophilum \\
\hline
\end{tabular}

Fuente: autores

Entre las bacterias BAL probióticas más estudiadas, encontramos a Lactobacillus rhamnosus. Es una bacteria que cumple con los estándares de las Naciones Unidas y con los requisitos clínicos, según documentación del ensayo FAO / OMS de 20017'. Esta bacteria presenta una alta tolerancia en condiciones de acidez, por lo que prevalece en el estómago, sobrevive al tránsito intestinal y es capaz de adherirse a la mucosa colónica humana, para luego 


\section{Mayo - Agosto}

ser capaz de colonizar el tracto gastrointestinal, después del respectivo tratamiento $0^{6,40}$. La evidencia existente reporta efectos benéficos del $L$. rhamnosus en la prevención y el tratamiento de la diarrea asociada a antibióticos, la diarrea por rotavirus, trastornos gastrointestinales e infecciones en las vías respiratorias en los niños, e inhibe el crecimiento y la adhesión de enteropatógenos ${ }^{45}$.

Tabla 2. Subespecies de Bifidobacterium reconocidas como probióticos

\begin{tabular}{|l|}
\hline B. animalis (subsp. animalis y lactis) \\
\hline B. pseudolongum (subsp. globosum y pseudolongum) \\
\hline $\begin{array}{l}\text { B. hermacidophilum (subsp. thermoacidophilum y } \\
\text { porcinum) }\end{array}$ \\
\hline B. longum (subsp. longum, infantis y suis) \\
\hline
\end{tabular}

Fuente: autores.

Bifidobacterium se destaca por ser una bacteria probiótica que también posee reconocimiento mundial a nivel científico y soporte documentado mediante la guía de la $\mathrm{FAO} / \mathrm{WHO}^{17}$, siendo un importante grupo de cepas probióticas comúnmente utilizadas en los productos lácteos fermentados ${ }^{46}$. Son gram-positivas, anaerobias, no móviles y sin esporulación. Las especies incluidas en el género Bifidobacterium ${ }^{47}$ se pueden observar en la tabla $1 \mathrm{y}$ tabla $2^{2,3}$.

El pH óptimo para el crecimiento de las Bifidobacteria está entre 6,0 y 7,0, y prácticamente ninguna puede crecer por debajo de $\mathrm{pH}$ 4,5 o por encima de 8,5. La temperatura óptima de crecimiento está entre 37 a y $41^{\circ} \mathrm{C}$, la mínima entre $25-28{ }^{\circ} \mathrm{C}$ y la máxima entre 43 a $45^{\circ} \mathrm{C}^{48}$. Algunos de los cultivos de Bifidobacterium más utilizadas como probióticos son $B$. adolescentis, B. longum, B. infantis y B. breve. Para el caso de la estabilidad de las bifidobacterias en el yogur, investigado por Underwood et al., se informa que varios ingredientes, como la cisteína de la proteína del suero de la leche y la caseína hidrolizadas, son eficaces para mantener su viabilidad en este. Así mismo, es vital el uso de bacterias lácticas como iniciadores de su producción, para intensificar la estabilidad de la bifidobacteria ${ }^{36}$. Por otro lado, Ferreira et al. reporta que las Bifidobacterium, particularmente la B. animalis ssp lactis, es más resistente contra el estrés ambiental y más estable en yogur, en comparación a la Bifidobacterium longum, Bifidobacterium breve y Bifidobacterium bifidum, que son bifidobacterias humanas, lo cual ha conllevado a que la B. animalis se utilice mayormente en los productos lácteos fermentados del mercado actual ${ }^{49}$.
Los probióticos: alimentos funcionales para lactantes

\section{Factores fisicoquímicos que afectan el desempeño de un probiótico}

Factores como el tipo de la cepa, la actividad del agua, la sensibilidad al oxígeno, la temperatura y el almacenamiento son también muy influyentes; debido a esto, no todas las cepas pueden ser fácilmente fabricadas ni comercializadas industrialmente ${ }^{50,51,52}$. Para que un probiótico pueda ser producido a nivel industrial, éste debe presentar un valor alto de crecimiento microbiano viable ${ }^{24}$. Por consiguiente, una medida de la concentración de la cepa en UFC determina los efectos mínimos en salud, lo que explica que ya existan referencias en la literatura de estos datos: por ejemplo, para la leche acidophilus se exige una concentración de 1X107 UFC de probiótico por $\mathrm{mL}$, para lograr el efecto esperado3,53. Así mismo, otro factor que afecta a los probióticos es la temperatura, pues varios estudios reportan que trabajar por debajo de $45^{\circ} \mathrm{C}$ propicia la supervivencia de las bacterias ${ }^{2,12,48}$.

Otro aspecto muy importante es la formulación de la matriz ${ }^{54}$, ya que, desde el punto de vista tecnológico, es la que más puede afectar la funcionalidad de un probiótico $3,47,55$. Puesto que el desempeño de los probióticos con efecto funcional es bien conocido en leche, quesos y helados lácteos ${ }^{56,57}$, pero poco en matrices tales como en cereales, avena, confitería y frutas, estas últimas implican un gran reto ${ }^{22,39}$. Por consiguiente, para mejorar el desempeño de diversos probióticos en estas nuevas matrices, se viene aplicando la encapsulación o microencapsulación como sustituto tecnológico ${ }^{58,59}$.

También se reportan estudios donde se ha llegado a analizar el comportamiento de tales matrices y su actividad probiótica desde la producción de sustancias por parte de estos, entre las que se encuentran las enzimas como las a-galactosidasas ${ }^{12}$. Igualmente, se ha observado, para el caso de tres especies diferentes de banano, que cada una presenta sustancias nutricionales tales como prebióticos ${ }^{60}$, componentes que proporcionan alimento y que pueden beneficiar más a un probiótico que a otro, favoreciendo por consiguiente la actividad prebiótica y los carbohidratos requeridos para su viabilidad ${ }^{61 .}$

La Microencapsulación se ha empleado como una metodología que beneficia la viabilidad y la estabilidad del probiótico en el alimento, debido a que encapsula el probiótico con una cubierta de aditivo químico grado alimento y de naturaleza química 
afín al alimento en el que va a ser incorporado ${ }^{62,63}$, lo que favorece la biodisponibilidad en el organismo específicamente en el tracto gastrointestinal7 $73,61,64$. La misión principal de la microencapsulación es proteger de la oxidación, humedad y los cambios bruscos de temperatura al probiótico durante largos periodos de almacenamiento9,59,65.

\section{Mecanismos de acción de los probióticos con efecto benéfico para la salud}

A pesar de todos los efectos funcionales de muchos probióticos, la forma como ellos actúan no está actualmente clara35; sin embargo, algunos estudios reportan los mecanismos de acción por los cuales se pueden dar los efectos benéficos: por adherencia y colonización en el intestino, y estimulación en la producción de sustancias mucosas, de manera que se fortalecen las uniones intestinales y se inhibe la translocación bacteriana ${ }^{27}$, por supresión del crecimiento de la bacteria patógena, por producción de sustancias antimicrobianas, por modulación del crecimiento de la microbiota, por producción de sustancias tóxicas, por reducción del pH intraluminal y por exclusión competitiva de bacterias patógenas de sitios de enlace ${ }^{11,35,37,38}$

\section{Bacillus coagulans, probiótico empleado por la industria de alimentos}

Este microorganismo probiótico fue aislado de la malta verde, en 1932, por los científicos HorowitzWlassowa y Nowotelnow ${ }^{53}$. Luego, científicos japoneses lo denominaron Lactobacillus sporogenes, pero, posteriormente, fue clasificado como Bacillus Coagulans. Este probiótico es una bacteria gram positiva, ácido láctica formadora de esporas; crece en un rango de temperatura entre 35 a $50^{\circ} \mathrm{C}$ y su rango de pH óptimo está entre 5.5 y $6.5^{66,67}$. La formación de esporas de este probiótico permite que sea resistente a los ácidos gástricos y a la bilis para llegar intacto al intestino grueso, gracias a la cápsula que lo protege ${ }^{53,68}$.

Los beneficios en salud del Bacillus coagulans reportados van desde mejoras en el tratamiento de la hiperlipidemia, la adenovirus, la influenza, la estomatitis aftosa y la caries dental, hasta tratamientos para la artritis ${ }^{50,69}$. Estudios clínicos reportan efectos benéficos en el dolor de estómago de niños, con una mezcla de Bacillus coagulans y prebióticos tales como los FOS (Fructooligosacáridos) $^{14,51}$; además, también se ha visto su efecto favorable en el tratamiento de la diarrea infantil|51,70,71 y de la hipercolesterolemia ${ }^{72}$.

El Bacillus coagulans es un probiótico potencial en alimentos funcionales debido a su capacidad para soportar altas temperaturas, y su formación de esporas lo hace más apto para trabajar en matrices alimentarias basadas en frutas, vegetales, cereales o granos ${ }^{61}$. Por consiguiente, este probiótico se hace atractivo en el amplio espectro del ámbito de la industria alimentaria, en su línea funcional $65,68,73$. Lo anterior brinda una oportunidad de trabajar los probióticos en alimentos funcionales dirigidos hacia la población infantil.

\section{Probióticos empleados en la producción de alimentos para bebé}

Conocidos los efectos en salud de los probióticos para población adulta, se despliega una vertiente en alimentos orientada hacia la línea de alimentos para niños, con un mayor énfasis en la producción de alimentos para bebes lactantes entre los 6 y 12 meses.

Estudios realizados en población infantil, exaltan los efectos positivos y mejoras en el tratamiento infecciones gastrointestinales y cólicos, con alimentos y suplementos que contenían un probiótico o mezclas de al menos dos probióticos ${ }^{74}$. Es así como, un estudio de Onubi y Poobalan, reportan que Sarawal et al. (2010), para inspeccionar el beneficio de los probióticos en el aumento de la talla y el peso, adicionó bifidobacterium lactis en una fórmula a base de leche para bebes de más de seis meses, y observó un efecto en el aumento de peso. Efectos similares se han encontrado con la adición de especies de Lactobacillus ${ }^{64,75}$

Las principales características de los alimentos suplementados con probióticos para esta población estaban adicionadas a leche, yogurt o a un alimento con origen natural fermentado ${ }^{75}$. Dentro de esta gama de propuestas, se observó poca exploración de probióticos en productos a base de frutas, las cuales también podrían proveer un excelente medio ambiente para estos microorganismos benéficos, debido a los nutrientes, contenido de carbohidratos y proteínas.

Se ha comprobado que la leche materna contiene una microbiota compuesta tanto por probióticos como prebióticos propios; por esto, es importante 


\section{Mayo - Agosto}

el uso de probióticos en la alimentación infantil, especialmente cuando un bebé no puede ser amamantado, pues proveerle un alimento con un microorganismo que le dará la posibilidad de estimular su sistema inmunológico es una condición ideal para el lactante ${ }^{64}$

Estudios han desafiado esta propiedad con probióticos pertenecientes a la familia de las BAL en producto a base de leche o lácteos. Para productos no lácteos, se tiene el Bacillus coagulans, el cual, como otros, ya se encuentra catalogado como alimento Generalmente Reconocido como Seguro en alimentos (GRAS), luego de ser aprobado y cumplir los requerimientos de la Guidelines for the Evaluation of Probiotics in Food de la FAO and WHO. Sin embargo, a este último se le requieren más pruebas reproducibles, especialmente para la población lactante, pues es necesario tener claro si hay efectos secundarios que afecten la salud y el adecuado desarrollo de este. A la fecha, no se ha reportado un caso negativo relacionado con un probiótico catalogado como GRAS'3; No obstante, organismos expertos en alimentación infantil, como el comité de la Sociedad Europea de Gastroenterología, Hepatología y Nutrición Pediátrica (ESPGHAN), han insistido en manifestar que los estudios reportados no inspiran mucha confianza, por encontrar incongruencias entre los resultados estadísticos ${ }^{52,75}$

Por otro lado, el comité ESPGHAN consideró pertinente declarar que hay que tener en cuenta que, en un bebé recién nacido, su microbiota no se encuentra todavía bien desarrollada; por lo cual la asimilación de una formulación con probióticos podría ser contraproducente antes del tiempo normalmente estipulado ${ }^{75}$.

Igualmente, ESPGHAN propone expandir el espectro del efecto benéfico en salud de los probióticos en lactantes hacia aspectos como el aumento en la talla de crecimiento del bebé, o hacia sintomatologías relacionadas con infecciones gastrointestinales, cólicos, y al no uso de antibióticos para bebé14. Todo apunta hacia la erradicación de conclusiones en donde los beneficios de un probiótico se replicarían a los demás probióticos semejantes de su género.

Sin embargo, aunque entre líneas es una especie de veto hacia estos intentos de innovación alimentaria
Los probióticos: alimentos funcionales para lactantes

en alimentos para bebé, lo que este comité expresa es que, mientras un producto suplementado con probióticos para bebé este bien certificado y apoyado sobre las evidencias científicas y estudios estipulados que pide un correcto Health Claim, se puede promover y comercializar ese producto ${ }^{75}$.

El éxito de un alimento certificado con probióticos para bebé dependerá del contexto en que sea puesto a prueba. Es por consiguiente que el estudio de Ojocheneme y Poobalam ${ }^{74}$, en defensa de la adición de probióticos, argumentan y exaltan que normalmente se acentúa el beneficio de estos resultados hacia la conservación de las defensas inmunológicas. En vista de esto, decidieron orientarse hacia el estudio de los efectos en el crecimiento y tamaño que podría llegar a alcanzar un bebé suplementado, es decir, una población infantil compuesta por bebes bien nutridos ${ }^{14,64,76,77,78,79}$.

Más que evitar el uso de probióticos en alimentos suplementados para bebé, el comité ESPGHAN y otros entes reguladores de igual importancia proponen, que los estudios sean de más largo plazo; en los cuales se observe el día a día de su consumo y sus efectos 75 ; queda lo anterior como la actual premisa para el debido proceso de un Health claim.

\section{Conclusiones}

Las bacterias ácido lácticas y las bifidobacterias son las de mayor trayectoria y uso actualmente en la preparación de alimentos funcionales de origen lácteo para lactantes. Por otro lado, sigue siendo materia de exploración el estudio de los diferentes probióticos, como el B. coagulans, en matrices tipo frutas, vegetales y granos, entre otros, para consumo en la nutrición de lactantes.

Aunque actualmente se ha avanzado a gran escala sobre el conocimiento de los probióticos en productos lácteos y su beneficio en salud, todavía hay cuestionamientos retadores y desafiantes como los relativos a la viabilidad y biodisponibilidad, según los diferentes entes reguladores.

Reconociendo los beneficios de los probióticos en la salud de los lactantes, su aplicación exige mayor cumplimiento de criterios de la Sociedad Europea de Gastroenterología, Hepatología y Nutrición Pediátrica. 


\section{Referencias bibliográficas}

1. Mani-López E, Palou E, López-Malo a. Probiotic viability and storage stability of yogurts and fermented milks prepared with several mixtures of lactic acid bacteria. J Dairy Sci. 2014;97(5):2578-90.

2. Sharma Shiwani, Anshul dutt Sharma BC. Functional Food : A Review. J Pharm Res. 2011;4(10):3618-9.

3. Ye W, Chen J, Wang J, Cai Y. The stability of probiotics added to the food. J Chem Pharm Res. 2013;5(12):27-9.

4. Maja B, Marica R, Ljiljana M, Svetlana N, Maja V-S, Aleksandra $\mathrm{D}-\mathrm{V}$. Improvement of production performance of functional fermented whey-based beverage. Chem Ind Chem Eng Q. 2012;20(00):96-96.

5. Walsh H, Cheng J, Guo M. Effects of Carbonation on Probiotic Survivability, Physicochemical, and Sensory Properties of MilkBased Symbiotic Beverages. J Food Sci. 2014;79(4).

6. Mpofu A, Linnemann AR, Sybesma W, Kort R, Nout MJR, Smid EJ. Development of a locally sustainable functional food based on mutandabota, a traditional food in southern Africa. J Dairy Sci. 2014;97(5):2591-9.

7. Das A, Ray S, Raychaudhuri U, Chakraborty R. Microencapsulation of Probiotic Bacteria and its Potential Application in Food Technology. Int J Agric Environ Biotechnol. 2014;7(1):47.

8. Bernardeau M, Vernoux JP. Overview of differences between microbial feed additives and probiotics for food regarding regulation, growth promotion effects and health properties and consequences for extrapolation of farm animal results to humans. Clin Microbiol Infect. 2013;19(4):321-30.

9. Sousa S, Gomes AM, Pintado MM, Silva JP, Costa P, Amaral $\mathrm{MH}$, et al. Characterization of freezing effect upon stability of, probiotic loaded, calcium-alginate microparticles. Food Bioprod Process. 2015;93:90-7.

10. Miyake Y, Yamamoto K. Role of gut microbiota in liver diseases. Hepatol Res. 2013;43(2):139-46.

11. Hickey L, Jacobs SE, Garland SM. Probiotics in neonatology. J Paediatr Child Health. 2012;48(9):777-83.

12. Liu X, Champagne CP, Lee BH, Boye JI, Casgrain M. Thermostability of Probiotics and Their $\alpha$-Galactosidases and the Potential for Bean Products. Biotechnol Res Int. 2014;2014:472723.

13. Van den Nieuwboer M, Brummer RJ, Guarner F, Morelli L, Cabana M, Claassen E. Safety of probiotics and synbiotics in children under 18 years of age. Benef Microbes. 2015;6(5):615-30.

14. Ackerberg TS, Labuschagne IL, Lombard MJ. The use of prebiotics and probiotics in infant formula. South African Fam Pract. 2012;54(4):322-3.

15. F A O Foodnutrition. Probiotics in food FOOD AND NUTRITION. FAO FOOD NUR Pap. 2006;

16. Tintore M. , Colome G., Santas J. EJ. Gut Microbiota Dysbiosis and Role of Probiotics in Infant Colic. Arch Clin Microbiol. 2017;8(4 (56)):1-7.

17. Ganguly NK, Bhattacharya SK, Sesikeran B, Nair GB, Ramakrishna BS, Sachdev HPS, et al. ICMR-DBT Guidelines for evaluation of probiotics in food. Indian J Med Res. 2011;134(7):22-5.

18. Araya M, Morelli L, Reid G, Sanders ME, Stanton C, Pineiro M, et al. Guidelines for the Evaluation of Probiotics in Food. Jt FAO/ WHO Work Gr Rep Draft Guidel Eval Probiotics Food. 2002;1-11.

19. Parlamento Europeo. Reglamento (UE) 2015/2283. D Of la Unión Eur Agencia Estatal Boletín Of del Estado. 2015;327:1-22.

20. Donovan SM, Schneeman B, Gibson GR, Sanders ME. Establishing and Evaluating Health Claims for Probiotics 1,2. Adv Nutr. 2012;3:723-5.

21. De Azevedo MSP, Innocentin S, Dorella F a., Rocha CS, Mariat $\mathrm{D}$, Pontes DS, et al. Immunotherapy of allergic diseases using probiotics or recombinant probiotics. J Appl Microbiol. 2013;115(2):319-33.

22. Martín-Muñoz MF, Fortuni M, Caminoa M, Belver T, Quirce S, Caballero T. Anaphylactic reaction to probiotics: Cow's milk and hen's egg allergens in probiotic compounds. Pediatr Allergy Immunol. 2012;23(8):778-84.

23. Hussey S, Wall R, Gruffman E, O’Sullivan L, Ryan CA, Murphy $\mathrm{B}$, et al. Parenteral antibiotics reduce bifidobacteria colonization and diversity in neonates. Int J Microbiol. 2011;2011.

24. Ismail IH, Licciardi P V., Tang MLK. Probiotic effects in allergic disease. J Paediatr Child Health. 2013;49(9):709-15.
25. Yang G, Liu Z-Q, Yang P-C. Treatment of allergic rhinitis with probiotics: an alternative approach. N Am J Med Sci. 2013;5(8):465-8.

26. Athalye-jape G, Deshpande G, Rao S, Patole S. Benefits of probiotics on enteral nutrition in preterm neonates : 2014;(1):1508-19

27. Oswari H, Prayitno L, Dwipoerwantoro PG, Firmansyah A, Makrides M, Lawley B, et al. Comparison of stool microbiota compositions, stool alpha1-antitrypsin and calprotectin concentrations, and diarrhoeal morbidity of Indonesian infants fed breast milk or probiotic/prebiotic-supplemented formula. J Paediatr Child Health. 2013;49(12):1032-9.

28. Li D, Rosito G, Slagle T. Probiotics for the prevention of necrotizing enterocolitis in neonates: an 8-year retrospective cohort study. J Clin Pharm Ther. 2013;38(6):445-9.

29. Closa-Monasterolo R, Gispert-Llaurado M, Luque V, Ferre N, Rubio-Torrents C, Zaragoza-Jordana M, et al. Safety and efficacy of inulin and oligofructose supplementation in infant formula: Results from a randomized clinical trial. Clin Nutr. Elsevier Ltd; 2013;32(6):918-27.

30. De Silva D, Geromi M, Panesar SS, Muraro a, Werfel T, HoffmannSommergruber K, et al. Acute and long-term management of food allergy: systematic review. Allergy . 2014;69(2):159-67.

31. Muller C, Busignies V, Mazel V, Forestier C, Nivoliez A, Tchoreloff P. Mechanistic approach to stability studies as a tool for the optimization and development of new products based on $\mathrm{L}$. rhamnosus Lcr35 ${ }^{\circledR}$ in compliance with current regulations. PLoS One. 2013;8(11):1-7.

32. Rodes L, Paul A, Coussa-Charley M, Al-Salami H, TomaroDuchesneau C, Fakhoury M, et al. Transit Time Affects the Community Stability of Lactobacillus and Bifidobacterium Species in an In Vitro Model of Human Colonic Microbiotia. Artif Cells, Blood Substitutes Biotechnol. 2011;39(6):351-6.

33. Jain D, Chaudhary HS. Clinical significance of probiotics in human. Int J Nutr Pharmacol Neurol Dis. 2014;4(1):11-22.

34. Rodrigues F, Sarmento B, Andrade J, Oliveira B. Review: Can microencapsulation be a means to increase survival of probiotics in cheese? Int J Probiotics Prebiotics. 2012;7(2):65-80.

35. Nauta et. al. Relevance of pre- and postnatal nutrition to development and interplay between themicrobiota and metabolic and immune systems. Am J Clin Nutr. 2013;98:586S - 593S.

36. Underwood $M$ a, German JB, Lebrilla CB, Mills D a. Bifidobacterium longum subspecies infantis: champion colonizer of the infant gut. Pediatr Res. 2015;77(0):229-35.

37. Fox M, Ahuja K, Eri R. Efficacy of Probiotics in the Prevention of Antibiotic-Associated Diarrhoea (Aad) in Children-a Review. J Probiotics. 2013;8(2):61-6.

38. Mugambi MN, Musekiwa A, Lombard M, Young T, Blaauw R. Synbiotics, probiotics or prebiotics in infant formula for full term infants: a systematic review. Nutr J. 2012;11(1):81.

39. Okamoto A;, Filho A. Probiotics: Reality of Benefits for Life, A Review. Int J Probiotics Prebiotics. 2016;11(1):7-14.

40. Bull M, Plummer S, Marchesi J, Mahenthiralingam E. The life history of Lactobacillus acidophilus as a probiotic: a tale of revisionary taxonomy, misidentification and commercial success. FEMS Microbiol Lett [Internet]. 2013;349(2):77-87.

41. Papadimitriou K, Zoumpopoulou G, FolignÃ® B, Alexandraki V, Kazou M, Pot B, et al. Discovering probiotic microorganisms: in vitro, in vivo, genetic and omics approaches. Front Microbiol. 2015;6(February):1-29.

42. Shoji. Viability of L. acidophilus microcapsules and their application to buffalo milk yoghurt. Food Bioprod Process. 2013;91:83-8.

43. Hanchi H, Hammami R, Kourda R, Hamida JB, Fliss I. Bacteriocinogenic properties and in vitro probiotic potential of enterococci from Tunisian dairy products. Arch Microbiol. 2014;196(5):331-44.

44. Oladipo IC, Sanni A, Swarnakar S. Phenotypic and Genomic Characterization of Enterococcus Species from Some Nigerian Fermented Foods. Food Biotechnol. 2013;27(1):39-53.

45. Vincenzetti S, Savini M, Cecchini C, Micozzi D, Carpi F, Vita A, et al. Effects of Lyophilization and Use of Probiotics on Donkey's Milk Nutritional Characteristics. Int J Food Eng. 2011;7(5).

46. Merenstein D, Gonzalez J, Young a G, Roberts RF, Sanders ME, Petterson S. Study to investigate the potential of probiotics in children attending school. Eur J Clin Nutr. 2011;65(4):447-53. 


\section{Mayo - Agosto}

47. Ozyurt Hazal OS. Properties of Probiotics and encapsulated probiotics in Food. Acta Sci Pol. 2014;13(4):413-24.

48. Celik OF, O'Sullivan DJ. Factors influencing the stability of freezedried stress-resilient and stress-sensitive strains of bifidobacteria. J Dairy Sci. 2013;96(6):3506-16.

49. Ferreira CL, Salminen S, Grzeskowiak L, Brizuela MA, Sanchez L, Carneiro H, et al. Terminology concepts of probiotic and prebiotic and their role in human and animal health. Rev Salud Anim. 2011;33(3):137-46.

50. Riazi S, Dover SE, Chikindas ML. Mode of action and safety of lactosporin, a novel antimicrobial protein produced by Bacillus coagulans ATCC 7050. J Appl Microbiol. 2012;113(3):714-22.

51. Saneian H, Pourmoghaddas Z, Roohafza H, Gholamrezaei A. Synbiotic containing Bacillus coagulans and fructooligosaccharides for functional abdominal pain in children. Gastroenterol Hepatol from Bed to Bench. 2015;8(1):56-65.

52. Vandenplas Y, Veereman-Wauters G, Greef E De, Peeters S, Casteels A, Mahler T, et al. Probiotics and prebiotics in prevention and treatment of diseases in infants and children. J Pediatr (Rio J). 2011;87(4):292-300.

53. Jurenka JS. Bacillus coagulons. Altern Med Rev. 2012;17(1):7681.

54. Arena MP, Caggianiello G, Fiocco D, Russo P, Torelli M, Spano $\mathrm{G}$, et al. Barley B-glucans-containing food enhances probiotic performances of beneficial bacteria. Int J Mol Sci. 2014;15:302539.

55. Rodrigues F, Sarmento B, Andrade J, Oliveira B. Review: Can microencapsulation be a means to increase survival of probiotics in cheese? Int J Probiotics Prebiotics. 2012;7(2):65-80.

56. Costa MP, Balthazar CF, Franco RM, Marsico ET, Cruz a G, Conte Junior $\mathrm{C}$ a. Changes on expected taste perception of probiotic and conventional yogurts made from goat milk after rapidly repeated exposure. J Dairy Sci. 2014;97(5):2610-8.

57. Strahinid I, Lukid J, Terzic-vidojevié A. Use of Lactobacillus helveticus BGRA43 for Manufacturing Fermented Milk Products. 2013;52(2):257-65.

58. Pedroso DL, Dogenski M, Thomazini M, Heinemann RJB, FavaroTrindade CS. Microencapsulation of Bifidobacterium animalis subsp. lactis and Lactobacillus acidophilus in cocoa butter using spray chilling technology. Braz J Microbiol. 2013;44(3):777-83.

59. Thantsha MS, Labuschagne PW, Mamvura CI. Supercritical CO2 interpolymer complex encapsulation improves heat stability of probiotic bifidobacteria. World J Microbiol Biotechnol. 2014;30(2):479-86.

60. Meyer R, Shah N. The role of pre- and probiotics in infant nutrition. J Fam Health Care. 2014;23(7):25-9.

61. Srisuvor N, Prakitchaiwattana C, Chinprahast N, Subhimaros S. Use of banana pur? ee from three indigenous Thai cultivars as food matrices for probiotics and application in bio-set-type yoghurt production. Int J Food Sci Technol. 2013;48:1640-8.

62. Nazzaro F, Orlando P, Fratianni F, Coppola R. Microencapsulation in food science and biotechnology. Curr Opin Biotechnol. Elsevier Ltd; 2012;23(2):182-6.

63. Ayama H, Sumpavapol P, Chanthachum S. Effect of encapsulation of selected probiotic cell on survival in simulated gastrointestinal
Los probióticos: alimentos funcionales para lactantes

tract condition. Songklanakarin J Sci Technol. 2014;36(3):291-9.

64. Tambakhe M.K PP. Supplementation of Infant Formula with Probiotics, Prebiotics, DHA \& ARA: A Systematic Review. J Food Nutr Sci. 2014;2(4):185.

65. Keller D, Farmer S, McCartney A, Gibson G. Bacillus coagulans as a probiotic. Food Sci Technol Bull Funct Foods. 2010;7:103-9.

66. $\mathrm{Xu}$ Y, Wang Y, Lin J. Use of Bacillus coagulans as a Dietary Probiotic for the Common Carp, Cyprinus carpio. J World Aquac Soc. 2014;45(4):403-11.

67. Honda H, Hoyles L, Gibson G, Farmer S. Impact of ganedenBC Bacillus Coagulans GBI-30 , 6086 ) on population dynamics of the human gut microbiota in a continuous culture fermentation system. Int J Probiotics Prebiotics. 2011;6(1):65-72.

68. Pandey KR, Shinde PS, Vakil B V. Evaluation of molecular variations in Probiotic Bacillus coagulans and its bacteriophage resistant mutants. Int J Curr Microbiol Appl Sci. 2015;4(4):34355.

69. Bora PS, Puri V, Bansal AK. Physicochemical properties and excipient compatibility studies of probiotic Bacillus coagulans spores. Sci Pharm. 2009;77(3):625-37.

70. Dutta P, Mitra U, Dutta S, Rajendran K, Saha TK, Chatterjee MK. Randomised controlled clinical trial of Lactobacillus sporogenes (Bacillus coagulans), used as probiotic in clinical practice, on acute watery diarrhoea in children. Trop Med Int Heal. 2011;16(5):555-61.

71. Fijan S. Microorganisms with claimed probiotic properties: An overview of recent literature. Int J Environ Res Public Health. 2014;11(5):4745-67.

72. Sudha RM, Sunita M, Sekhar BM, Limited UB, Park SPB. Safety Studies of Bacillus Coagulans Unique IS-2 in rats : morphological , biochemical and clinical evaluations. Int J probiotics Prebiotics. 2011;6(1):43-8.

73. Sharma A., Sanjeev S. the Current Status of Probiotics - a Report From India. Int J Probiotics Prebiotics. 2015;10(2):47-52.

74. Onubi OJ, Poobalan AS, Dineen B, Marais D, McNeill G. Effects of probiotics on child growth: A systematic review. J Heal Popul Nutr. 2015;34(1)

75. Braegger C, Chmielewska A, Decsi T, Kolacek S, Mihatsch W, Moreno L, et al. Supplementation of infant formula with probiotics and/or prebiotics: A systematic review and comment by the ESPGHAN committee on nutrition. J Pediatr Gastroenterol Nutr. 2011;52(2):238-50.

76. Muhimbula HS, Issa-Zacharia A, Kinabo J. Formulation and sensory evaluation of complementary foods from local, cheap and readily available cereals and legumes in Iringa, Tanzania. African J Food Sci. 2011;5(1):26-31.

77. Lassen J, Yazdankhah S. Assessment of Probiotics in Infant Formula and Cereal Based Baby Foods Containing Bifidobacterium lactis Bb12- Update 2014. Eur J Nutr Food Saf. 2015;5(2):101-3.

78. Lestari LA, Setyabudi FMCS, Julia M, Amalia LD. Effect of Synbiotic Yogurt Made with Indigenous Probiotic Lactobacillus plantarum Mut7 and Sweet Potato Fiber ( Ipomoea batatas ) in Healthy Children. Int Res J Microbiol. 2013;4(3):98-102.

79. Labuschagne I, van Niekerk E, Lombard MJ. Acidified infant formula explained. South African Fam Pract. 2013;55(4):354-6. 